This item was submitted to Loughborough's Research Repository by the author.

Items in Figshare are protected by copyright, with all rights reserved, unless otherwise indicated.

\title{
An algorithm for detecting Directional Quasi-Convexity
}

PLEASE CITE THE PUBLISHED VERSION

LICENCE

CC BY-NC-ND 4.0

\section{REPOSITORY RECORD}

Dullin, Holger R., and Francesco Fasso. 2019. "An Algorithm for Detecting Directional Quasi-convexity". figshare. https://hdl.handle.net/2134/296. 


\title{
An algorithm for detecting Directional Quasi-Convexity
}

\author{
Holger Dullin * and Francesco Fassò ${ }^{\dagger}$
}

(December 22, 2002)

\begin{abstract}
Directional Quasi-Convexity (DQC) is a sufficient condition for Nekhoroshev stability, that is, stability for finite but very long times, of elliptic equilibria of Hamiltonian systems. The numerical detection of DQC is elementary for system with three degrees of freedom. In this article, we propose a recursive algorithm to test DQC in any number $n \geq 4$ of degrees of freedom.
\end{abstract}

\section{Introduction}

A. Lyapunov-stability of elliptic equilibria of Hamiltonian systems is often very difficult to establish. A weaker stability notion, that of Nekhoroshev-stability, has emerged over recent years and seems to be potentially of large impact for systems with $n \geq 3$ degrees of freedom. For systems with two degrees of freedom, instead, KAM theory can be used to prove Lyapunov stability, so we do not consider this case here. Roughly speaking, Nekhoroshev-stability [14] consists of the fact that motions starting very near the equilibrium remain close to it for very long times. Specifically, denoting by $d(t)$ the distance from the equilibrium at time $t$, the equilibrium is Nekhoroshev-stable if, for all sufficiently small $\epsilon$, for any motion one has

$$
d(0) \leq \epsilon \quad \Rightarrow \quad d(t) \leq c_{1} \epsilon^{a} \quad \text { for } \quad|t| \leq c_{2} \exp \left(\epsilon^{-b}\right)
$$

where $c_{1}, c_{2}, a$ and $b$ are positive constants independent of $\epsilon$. Typically, $a$ and $b$ are of the order of $1 / n$.

At first, Nekhoroshev stability was studied under the hypothesis that the frequencies of the equilibrium satisfy a strong nonresonance condition, typically a Diophantine one (see e.g. [9], [10]). This condition leads to a particularly simple approach but has the shortcoming that, if the frequencies depend on external parameters, then resonances are unavoidable. Because of this, more recently, the general problem has been reconsidered [6] [15] [11] [1] following an original proposal by Nekhoroshev [14].

Within such an approach, if the Hamiltonian is analytic and if the frequencies of the equilibrium are nonresonant up to order four, then sufficient conditions for Nekhoroshev-stability are deduced

\footnotetext{
*Loughborough University, Department of Mathematical Sciences, Loughborough, LE11 3TU, United Kingdom, h.r.dullin@lboro.ac.uk

${ }^{\dagger}$ Università di Padova, Dipartimento di Matematica Pura e Applicata, Via G. Belzoni 7, 35131 Padova, Italy, fasso@math. unipd.it
} 
from the analysis of the truncated fourth-order Birkhoff normal form

$$
\Omega \cdot I+\frac{1}{2} I \cdot M I
$$

Here $\Omega=\left(\Omega_{1}, \ldots, \Omega_{n}\right)$ is the frequency vector of the equilibrium, $M$ is a certain $n \times n$ symmetric matrix, and $I$ is the vector the entries of which are the $n$ action functions $I_{1}, \ldots, I_{n}$, i.e., in suitable canonical coordinates centered on the equilibrium,

$$
I_{j}=\frac{1}{2}\left(p_{j}^{2}+q_{j}^{2}\right), \quad j=1, \ldots, n .
$$

(For the reader's convenience, the construction of the normal form is recalled in the Appendix.) If all the components of $\Omega$ have the same sign, then the equilibrium is Lyapunov-stable. Sufficient conditions for Nekhoroshev-stability are instead either the convexity of the Birkhoff normal form, namely the definitness of the quadratic form $I \cdot M I$, or certain generalizations of it known as quasiconvexity [6] [15] [11] and as directional quasi-convexity [1]. The Birkhoff normal form is said to be

- Quasi-Convex, if the restriction of the quadratic form $I \cdot M I$ to the subspace orthogonal to $\Omega$ is definite, that is

$$
\Omega \cdot I=0 \quad \text { and } \quad I \cdot M I=0 \quad \Longrightarrow \quad I=0 .
$$

- Directionally Quasi-Convex (DQC), if

$$
\Omega \cdot I=0, \quad I \cdot M I=0 \text { and } I_{1}, \ldots, I_{n} \geq 0 \quad \Longrightarrow \quad I=0 .
$$

Quasi-convexity is a typical condition in the general Nekhoroshev theory, see e.g. [14], [2], [12]. The notion of DQC is instead specific to equilibria: it arises from the fact that, for an equilibrium, the action functions are non-negative, so that it is sufficient to check quasi-convexity only in the "first $2^{n}$-ant" of $\mathbb{R}^{n}$, where $I_{1} \geq 0, \ldots, I_{n} \geq 0$, see [1].

Nekhoroshev-stability of elliptic equilibria has been investigated so far in two classic problems. One is the triangular Lagrangian point L4 and L5 of the spatial restricted three body problem, for which $n=3$, see [1]. The other are the so called Riemann ellipsoids, for which $n=4$, see [7] [8]. In these cases, quasi-convexity is either never or just exceptionally encountered, while DQC ensures Nekhoroshev-stability for a large set of values of the parameters.

In order to prove Nekhoroshev-stability of elliptic equilibria which lack quasi-convexity or directional quasi-convexity, one should try to exploit the so called "steepness" properties [14]. However, steepness is much more difficult to test and leads to much worse stability estimates. Moreover, Nekhoroshev-stability of equilibria under a steepness condition has been so far proven only in a very special case with three degrees of freedom, so some theoretical work is still needed here. Therefore, it is in any case convenient to first test quasi-convexity and directional quasiconvexity.

B. In view of this, it is necessary to possess an algorithm for determining DQC. In fact, while the numerical verification of quasi-convexity is an elementary task, ${ }^{1}$ that of DQC is a priori not as easy, with the exception of the case $n=3$. For $n=3$ there is a very simple algorithm that has already been used in [1] and will be recalled here later, in Section 3.C. An algorithm for the case $n=4$, somewhat mimicked on that for $n=3$, was used in [7]. However, this algorithm reduced the

\footnotetext{
${ }^{1}$ See section 3.C.
} 


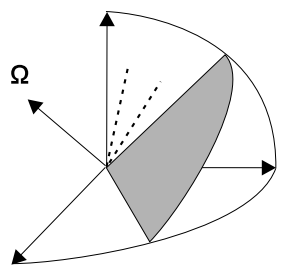

(a)

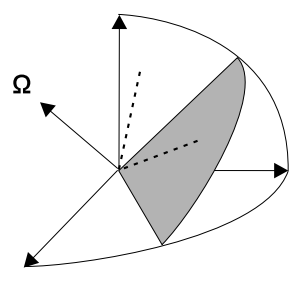

(b)

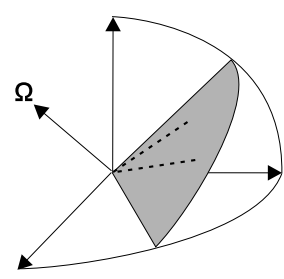

(c)

Figure 1: (a) DQC and (b,c) not DQC for $n=3$. The intersection of the plane orthogonal to $\Omega$ with $\mathbb{R}_{+}^{3}$ is shaded; the dotted lines represent two of the four rays forming $\mathcal{C}_{\Omega, M}$.

detection of DQC to checking for zeros of a function on the circle, which is a costly and delicate numerical operation. Moreover, such a procedure does not seem to be practically generalizable to higher dimensions.

The purpose of this paper is therefore that of providing an algorithm for detecting DQC in any number of degrees of freedom. The algorithm we propose is recursive: it reduces the problem to that of recursively testing DQC for systems with fewer degrees of freedom, until dimension 3 is reached and DQC can be tested directly.

In Section 2 we explain the idea of the method and prove the key result, that is, the recursion. The algorithm is then described in Section 3. Finally, in order to provide an example, in Section 4 we report very shortly on some partial (and not very encouraging, for what concerns the appearence of DQC) results for the magnetic top known as Levitron. This is modeled as a Hamiltonian system which, after reduction, has an elliptic equilibrium in five degrees of freedom, see [3] [4] and references therein.

\section{The recursion}

A. DQC. In this Section we explain the general idea of the method. To this end, we begin with some comments on DQC. We denote by $\mathrm{LS}_{n}$ the set of all $n \times n$ real symmetric matrices and

$$
\mathbb{R}_{+}^{n}:=\left\{x \in \mathbb{R}^{n}: x_{1} \geq 0, \ldots, x_{n} \geq 0\right\} .
$$

The DQC problem consists of the following:

(P1) Given a vector $\Omega \in \mathbb{R}^{n}$ and a symmetric matrix $M \in \mathrm{LS}_{n}$, determine whether the equations

$$
M x \cdot x=0, \quad \Omega \cdot x=0, \quad x \in \mathbb{R}_{+}^{n}
$$

have any nonzero solution. If yes, then $(\Omega, M)$ is not $D Q C$.

The equations $M x \cdot x=0$ and $\Omega \cdot x=0$ describe a cone ${ }^{2}$ in $\mathbb{R}^{n}$, which is obtained by interesecting the "quadratic" cone $M x \cdot x=0$ with the subspace $\Omega \cdot x=0$. DQC of $(\Omega, M)$ amounts to the fact that this cone intersects $\mathbb{R}_{+}^{n}$ only in the origin. Hence, considering the slightly modified cone

$$
\mathcal{C}_{\Omega, M}:=\left\{x \in \mathbb{R}^{n}: M x \cdot x=0, \Omega \cdot x=0, x \neq 0\right\}
$$

\footnotetext{
${ }^{2}$ We use the term "cone" in its broader term: a cone $C$ is a subset of $\mathbb{R}^{n}$ such that $\lambda C \subset C$ for all $\lambda>0$.
} 


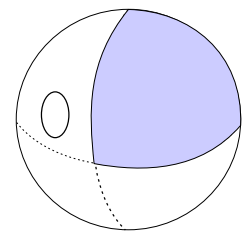

(a)

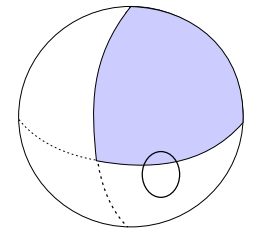

(b)

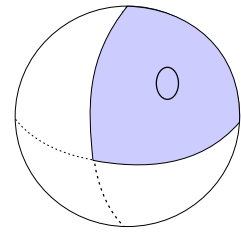

(c)

Figure 2: Intersection of the cone $\mathcal{C}_{\Omega, M}$ (drawn as a circle) and of $\mathbb{R}_{+}^{n}$ (shaded) with the unit sphere in the subspace orthogonal to $\Omega$, for $n=4$ : (a) DQC; (b) and (c) not DQC. The picture shows the projection of the subspace orthogonal to $\Omega$ onto one of the three-dimensional coordinate subspaces. Specifically, $\Omega$ is assumed to have one positive and three negative entries and the projection is onto the three-dimensional subspace of $\mathbb{R}^{4}$ spanned by the three negative entries. If any of the other three projections were used, then the shaded region would be a smaller spherical triangle. If $\Omega$ had two positive and two negative components, instead, then the shaded region would be of rectangular shape.

which is obtained by removing the origin from the previous one, DQC of $(\Omega, M)$ amounts to

$$
\mathcal{C}_{\Omega, M} \cap \mathbb{R}_{+}^{n}=\emptyset .
$$

Under suitable but generic conditions, $\mathcal{C}_{\Omega, M}$ is a smooth manifold of dimension $n-2$. For instance, it is sufficient to require

$$
\Omega \neq 0, \quad \operatorname{det} M \neq 0
$$

even though the latter condition can be slightly relaxed, as we will do later. There are a few trivial cases of DQC:

(T1) If the vector $\Omega$ has all entries of the same sign, then the subspace $\Omega \cdot x=0$ only intersect $\mathbb{R}_{+}^{n}$ in the origin and $(\Omega, M)$ is DQC for any $M$.

(T2) If $(\Omega, M)$ is quasi-convex, then $\mathcal{C}_{\Omega, M}$ is empty and $(\Omega, M)$ is DQC. (This includes convexity of $M$ as a special case.)

Figures 1 and 2 provide an illustration of DQC for $n=3$ and $n=4$; in both cases we assume (2.1) and we exclude the trivial cases T1 and T2. As it will be clear later, the case $n=3$ is somewhat special, but it is easy to visualize. In fact, the cone $M x \cdot x=0$ intersects the plane $\Omega \cdot x=0$ in two lines through the origin and hence $\mathcal{C}_{\Omega, M}$ consists of four rays. DQC amounts to the fact that none of them belongs to the positive octant $\mathbb{R}_{+}^{3}$, see Figure 1 . Thus, detection of DQC for $n=3$ reduces to the simple task of determining these rays.

In order to visualize the case $n=4$, which is a better representative of the general case, in Figure 2 we depict the three-dimensional subspace orthogonal to $\Omega$. Furthermore, exploiting the homogenity of the problem, we restrict everything to the unit sphere so as to simplify the picture. The cone $\mathcal{C}_{\Omega, M}$ intersects this sphere in a curve, drawn as a circle in the pictures. (In fact, the intersection with the subspace orthogonal to $\Omega$ is a two-dimensional cone, which in turn intersects the unit sphere in a one-dimensional cone). The intersection of $\mathbb{R}_{+}^{4}$ with this sphere is a twodimensional region, which is shaded in the picture. Thus, DQC amounts to the fact that the circle does not intersect the shaded region. 
B. The idea of the method. We can now explain the idea of the method for testing DQC for a system with any number $n \geq 4$ of degrees of freedom. We proceed as follows:

1. Consider all connected components of the cone $\mathcal{C}_{\Omega, M}$ and find a point in each of them.

2. If this point belongs to $\mathbb{R}_{+}^{n}$ then $(\Omega, M)$ is not DQC and hence the problem is solved.

3. Otherwise, we must determine whether the considered component of $\mathcal{C}_{\Omega, M}$ intersects $\mathbb{R}_{+}^{n}$. By connectedness, this may only happen if this component "enters" $\mathbb{R}_{+}^{n}$ through its boundary, that is, if it intersects one of the $(n-1)$-dimensional coordinate hyperplanes. Hence, the problem becomes:

(P2) For each connected component of $\mathcal{C}_{\Omega, M}$, does any of the $n$ systems of equations

$$
M x \cdot x=0, \quad \Omega \cdot x=0, \quad x_{j}=0, \quad x \in \mathbb{R}_{+}^{n}
$$

$(j=1, \ldots, n)$ have a nonzero solution? If yes, then $(\Omega, M)$ is not DQC.

As one immediately realizes, each problem (P2) is still of the form (P1), but for a pair $\left(\Omega^{j}, M^{j}\right) \in$ $\mathbb{R}^{n-1} \times \mathrm{LS}_{n-1}$ which is obtained by cancelling the $j$-th row from $\Omega$ and the $j$-th row and column from $M$. Hence, the problem can be studied by recursively checking DQC of pairs

$$
\left(\Omega^{j_{1} j_{2} \cdots}, M^{j_{1} j_{2} \ldots}\right) \in \mathbb{R}^{m} \times \mathrm{LS}_{m}, \quad m=n-1, n-2, \ldots
$$

The algorithm stops if, at some level, a nonzero intersection with $\mathbb{R}_{+}^{m}$ is found ( $\Rightarrow$ no DQC). Within the recursive tree, a branch is stopped if one of the trivial cases $\mathrm{T} 1$ and $\mathrm{T} 2$ is met, otherwise it goes on until dimension $m=3$ is reached, where DQC is tested directly.

C. The recursion. The critical element in this procedure is that one should be able to identify all connected components of the cones $\mathcal{C}_{\Omega, M}, \mathcal{C}_{\Omega^{j}, M^{j}} \ldots$ and to determine a point in each of them. This is not a problem because, as we now show, a cone of dimension $r \geq 3$ without its vertex either is connected or has two connected components.

In order to formalize this fact, we consider a pair $(\widehat{\Omega}, \widehat{M}) \in \mathbb{R}^{m} \times \mathrm{LS}_{m}, m \geq 4$, and make the following hypotheses:

(R1) The vector $\widehat{\Omega} \neq 0$

(R2) The restriction of the quadratic form $x \cdot \widehat{M} x$ to the subspace orthogonal to $\widehat{\Omega}$ is nondegenerate which together ensure that $\mathcal{C}_{\widehat{\Omega}, \widehat{M}}$ is a smooth manifold, and

(R3) $(\widehat{\Omega}, \widehat{M})$ is not quasi-convex

which ensures that $\mathcal{C}_{\widehat{\Omega}, \widehat{M}}$ is not empty, see T2. Thus, we have

Proposition 1 Assume that $(\widehat{\Omega}, \widehat{M}) \in \mathbb{R}^{m} \times \mathrm{LS}_{m}, m \geq 4$, satisfies conditions $R 1$, R2 and $R 3$. Then

i. $\mathcal{C}_{\widehat{\Omega}, \widehat{M}}$ is a smooth manifold which has either one or two connected components.

ii. If $\mathrm{C}_{\widehat{\Omega}, \widehat{M}}$ has two connected components, then they are transformed into each other by the reflection $x \mapsto-x$.

The proof of this fact is postponed to the following subsection. Here, we use it to formalize the recursive argument. In order to determine a point on each connected component of $\mathcal{C}_{\widehat{\Omega}, \widehat{M}}$ we use the fact that $\mathcal{C}_{\widehat{\Omega}, \widehat{M}}$ is symmetric under reflections and, if it is not connected, then its two components 
are symmetric to each other under reflection. Therefore, if we pick up a point on the cone and reflect it with respect to the origin, we obtain two points on the same component if the cone is connected (which does not harm), one point on each component if it is not connected. The case of interest is when none of these two points belong to $\mathbb{R}_{+}^{m}$, otherwise we are done. Thus, we assume

(R4) There exists a point $\bar{x} \in \mathcal{C}_{\widehat{\Omega}, \widehat{M}}$ such that $\bar{x} \notin \mathbb{R}_{+}^{m}$ and $-\bar{x} \notin \mathbb{R}_{+}^{m}$.

The recursive argument is now the content of the following Proposition 2 where, as before, we denote by $\widehat{\Omega}^{j}$ the vector obtained by cancelling the $j$-th row from a vector $\widehat{\Omega}$ and by $\widehat{M}^{j}$ the matrix obtained by cancelling the $j$-th row and the the $j$-th column from a matrix $M$.

Proposition 2 Assume that $(\widehat{\Omega}, \widehat{M}) \in \mathbb{R}^{m} \times \mathrm{LS}_{m}, m \geq 4$, satisfies all four conditions $R 1-R 4$. Then, $(\widehat{\Omega}, \widehat{M})$ is $D Q C$ if and only if all pairs $\left(\widehat{\Omega}^{j}, \widehat{M}^{j}\right)$ are $D Q C, j=1, \ldots, m$.

Proof As just explained, Hypothesis R4 ensures that each connected component of $\mathcal{C}_{\widehat{\Omega}, \widehat{M}}$ has a point outside $\mathbb{R}_{+}^{m}$. Since the cone $\mathcal{C}_{\widehat{\Omega}, \widehat{M}}$ is a smooth manifold, each of its connected components is a smooth connected manifold and hence is arcwise connected. Thus, one component intersects $\mathbb{R}_{+}^{m}$ if and only if it intersects at least one of the $(m-1)$-dimensional "faces" of $\mathbb{R}_{+}^{m}$, that is, if and only if at least one of the $m$ systems of equations

$$
\widehat{\Omega}^{j} \cdot x=0, \quad \widehat{M}^{j} x \cdot x=0, \quad x \in \mathbb{R}_{+}^{m-1} \quad(j=1, \ldots, m)
$$

has a nonzero solution. Since these equations cover both components at once, we conclude that $(\widehat{\Omega}, \widehat{M})$ is DQC if and only if none of the systems (2.3) has a nonzero solution, that is if and only if all pairs $\left(\widehat{\Omega}^{j}, \widehat{M}^{j}\right)$ are DQC.

Remark: The statement of the Proposition is not true for $m=3$ because in that case the connected components of $\mathcal{C}_{\widehat{\Omega}, \widehat{M}}$ are four rays which (generically) do not intersect the coordinate planes. This is why the recursion is stopped when dimension three is reached.

D. A Lemma on cones. We prove here Proposition 1. Since the restriction of a quadratic form to a subspace is still a quadratic form, $\mathcal{C}_{\widehat{\Omega}, \widehat{M}}$ is a quadratic cone. Hence, the proof of Proposition 1 essentially reduces to counting the connected components of a quadratic cone minus the origin. This is a simple exercise - we do it here because we could not find it anywhere.

Let us call definitness index of a quadratic form $y \cdot A y, A \in \mathrm{LS}_{r}$, the smallest integer between its positive and negative indexes of inertia, that is, the number

$$
k(A)=\min \left(n_{+}, n_{-}\right)
$$

where $n_{+}$is the number of positive eigenvalues of $A$ and $n_{-}$is the number of its negative eigenvalues. The quadratic form is definite if and only if its definitness index is zero, in which case the cone $\mathcal{C}_{A}$ is empty. Thus, we are only interested to the case $k(A) \geq 1$.

Lemma Let $A \in \mathrm{LS}_{r}, r \geq 2$, be non-singular and have definitness index $k \geq 1$. Then the set $\left\{y \in \mathbb{R}^{r}: y \cdot A y=0\right\}$ is a cone over $S^{k-1} \times S^{r-k-1}$.

Proof A cone "over" a subset $B$ of $\mathbb{R}^{r}$ is the union of all lines connecting the origin to the points of $B$. Hence, by homogeneity of the quadratic form $y \cdot A y$, the Lemma can be proved by showing that the intersection of the cone $y \cdot A y=0$ with the unit sphere $S^{r-1}$ in $\mathbb{R}^{r}$ is diffeomorphic to $S^{k-1} \times S^{r-k-1}$. We construct the diffeomorphism by a rotation which diagonalizes $A$ followed by a rescaling. Specifically, let us denote by $\pm \lambda_{1}, \ldots, \pm \lambda_{k}, \mp \lambda_{k+1}, \ldots, \mp \lambda_{r}$ the eigenvalues of $A$, with 
all $\lambda_{j}>0$ given that $\operatorname{det} A \neq 0$. (The double signs refer to the fact that $A$ has either $k$ positive or $k$ negative eigenvalues). Since $A$ is symmetric, there exists a matrix $Q \in \mathrm{SO}(r)$ such that

$$
Q^{T} A Q= \pm \operatorname{diag}\left(\lambda_{1}, \ldots, \lambda_{k},-\lambda_{k+1}, \ldots,-\lambda_{r}\right) .
$$

In the rescaled coordinates

$$
z=\operatorname{diag}\left(\lambda_{1}^{1 / 2}, \ldots, \lambda_{r}^{1 / 2}\right) Q^{T} y
$$

the cone is given by

$$
z_{1}^{2}+\ldots+z_{k}^{2}=z_{k+1}^{2}+\ldots+z_{r}^{2} .
$$

Its intersection with the sphere $\sum_{j=1}^{r} z_{j}^{2}=2$, which is diffeomorphic to its intersection with the unit sphere in the original coordinates $y$, is given by

$$
z_{1}^{2}+\ldots+z_{k}^{2}=1, \quad z_{k+1}^{2}+\ldots+z_{r}^{2}=1 .
$$

This is the product of two spheres $S^{k-1} \times S^{r-k-1}$.

The proof of Proposition 1 is an immediate application of the Lemma:

Proof of Proposition 1 Let us preliminarily write $\mathcal{C}_{\widehat{\Omega}, \widehat{M}}$ in the form of a quadratic cone. (This introduces some notations that will be used later). By hypothesis $\mathrm{R} 1$, there exists a rotation $R \in \mathrm{SO}(m)$ which carries $\Omega$ to any of the bases vector of $\mathbb{R}^{m}$, e.g. to $e_{m}=(0, \ldots, 0,1)^{T}$ :

$$
R^{T} \widehat{\Omega}=|\widehat{\Omega}| e_{m} .
$$

Denote by $A(\widehat{\Omega}, \widehat{M})$ the upper-left diagonal block of $R^{T} \widehat{M} R$, that is

$$
R^{T} \widehat{M} R=\left(\begin{array}{cc}
A(\widehat{\Omega}, \widehat{M}) & * \\
* & *
\end{array}\right)
$$

In the new coordinates $\left(\begin{array}{c}y \\ y_{m}\end{array}\right)=R^{T} x$, where $y \in \mathbb{R}^{m-1}$, the subspace orthogonal to $\widehat{\Omega}$ is given by $y_{m}=0$ and the restriction of the quadratic form $x \cdot \widehat{M} x$ to this subspace is $y \cdot A(\widehat{\Omega}, \widehat{M}) y$. Hence, the cone $\mathcal{C}_{\widehat{\Omega}, \widehat{M}}$ is diffeomorphic to the cone

$$
\mathcal{C}_{A(\widehat{\Omega}, \widehat{M})}=\left\{y \in \mathbb{R}^{m-1}: y \cdot A(\widehat{\Omega}, \widehat{M}) y=0, y \neq 0\right\} .
$$

Hypothesis R2 exactly means that the matrix $A(\widehat{\Omega}, \widehat{M})$ is nonsingular, while hypothesis $\mathrm{R} 3$ is equivalent to the fact that the definitness index $k(A) \geq 1$. Hence the Lemma implies that, for $r:=m-1 \geq 3$, the cone $\mathcal{C}_{A(\widehat{\Omega}, \widehat{M})}$ is diffeomorphic to $(0, \infty) \times S^{k-1} \times S^{r-k-1}$. Since $1 \leq k \leq r / 2$, we have $r-k-1 \geq 1$ for $r \geq 3$. Since $S^{0}=\left\{x \in \mathbb{R}: x^{2}=1\right\}$ consists of two points while all other spheres $S^{p}, p \geq 1$, are connected, it follows that $\mathcal{C}_{\widehat{\Omega}, \widehat{M}}$ is connected if $k>1$ and has two connected components if $k=1$.

When $k=1$, in the coordinates $z$ used in the proof of Lemma 2, the two components of $\mathcal{C}_{A(\widehat{\Omega}, \widehat{M})}$ are given by the two signs in

$$
z_{1}= \pm 1, \quad z_{2}^{2}+\ldots+z_{r}^{2}=1 .
$$

Hence, a point $z$ belongs to one of them if and only if $-z$ belongs to the other. It is evident that this property is inherited by the coordinates $y$ and, by linearity, it extends to $\mathcal{C}_{\widehat{\Omega}, \widehat{M}}$, too. 


\section{The algorithm}

A. The algorithm. Our algorithm is applicable to a pair $(\Omega, M) \in \mathbb{R}^{n} \times \operatorname{LS}_{n}$ under the following two hypotheses:

(H1) The vector $\Omega$ has no zero entries.

(H2) The restriction of $x \cdot M x$ to the subspace orthogonal to $\Omega$ is nondegenerate.

These hypotheses ensure that all vectors $\Omega^{j_{1} \ldots}$ and all matrices $M^{j_{1} \ldots}$ constructed by repeatedly cancelling rows and columns satisfy hypotheses $\mathrm{R} 1$ and $\mathrm{R} 2$. Thus, the algorithm reduces to recursively applying the procedure of Proposition 2, testing each time conditions R3 and R4.

Remark: Hypothesis H1 is a nonresonance condition on $\Omega$ which, in the present context, is not really restrictive: without it, the very existence of the Birkhoff normal form (1.2) would not be ensured (see the appendix). Hypothesis H1 and H2 play an essential role for the algorithm, since they ensure that $\mathcal{C}_{\Omega, M}$ and all other cones $\mathcal{C}_{\Omega^{j_{1} \cdots, M^{j_{1}} \ldots}}$ are smooth manifolds of the right dimensions. Hence, we do not see how to relax them. Of course, H2 is satisfied whenever $\operatorname{det} M \neq 0$.

The algorithm can be structured straightforwardly: Given $(\Omega, M)$, we first check H1 and H2. If they are satisfied, the following (recursive) procedure is applied to $(\Omega, M)$. The procedure takes a pair $(\widehat{\Omega}, \widehat{M}) \in \mathbb{R}^{m} \times \mathrm{LS}_{m}$ as argument and performs the following tasks:

1. Check the trivial DQC cases T1 and T2. (Note that T2 coincides with hypothesis R3 and must be checked for the recursion to be valid; testing $\mathrm{T} 1$ is just a matter of convenience.) If $(\widehat{\Omega}, \widehat{M})$ is found to be DQC, the procedure exits.

2. If $m=3$, the procedure calls the three-dimensional algorithm (see below) to detect whether $(\widehat{\Omega}, \widehat{M})$ is DQC. If $(\widehat{\Omega}, \widehat{M})$ is found to be not DQC, then the procedure returns "Non-DQC" and exits (and the algorithm is stopped). Otherwise, the procedure exits.

3. The procedure finds a point $\bar{x} \in \mathrm{C}_{\widehat{\Omega}, \widehat{M}}$ (see below). If this point violates $\mathrm{R} 4$, then $(\widehat{\Omega}, \widehat{M})$ is not DQC and hence the procedure returns "Non-DQC" and exits (and the algorithm is stopped).

4. The procedure calls itself on all pairs $\left(\widehat{\Omega}^{j}, \widehat{M}^{j}\right)$.

If, at any level, the procedure returns "Non-DQC" then $(\Omega, M)$ is not DQC and hence the algorithm is stopped. If "Non-DQC" is never returned during the whole recursion, then $(\Omega, M)$ is DQC.

The implementation of the algorithm is also straightforward, so we limit ourselves to a few remarks. Testing H2 can be done simply by computing the eigenvalues of the matrix $A(\Omega, M)$ as in (2.6). The rotation $R$ leading to such a matrix can be constructed e.g. by a Gram-Schmidt. Condition R3 is tested similarly.

The point $\bar{x}$ which appears in $\mathrm{R} 4$ can be computed in a variety of ways. For instance, considering again the the matrix $R$ as in $(2.5)$ and denoting by $Q$ the matrix which casts the matrix $A(\widehat{\Omega}, \widehat{M})$ as in (2.6) into the diagonal form (2.4), then

$$
\bar{x}=R\left(\begin{array}{c}
Q \bar{y} \\
0
\end{array}\right), \quad \bar{y}=\left(\lambda_{m-1}^{1 / 2}, 0, \ldots, 0, \lambda_{1}^{1 / 2}\right)^{T} .
$$

It only remains to describe the:

Algorithm for $m=3$ : If $(\widehat{\Omega}, \widehat{M}) \in \mathbb{R}_{+}^{3} \times \mathrm{LS}_{3}$ satisfies hypothesis $\mathrm{R} 1, \mathrm{R} 2$ and $\mathrm{R} 3$ then $\mathcal{C}_{\widehat{\Omega}, \widehat{M}}$ consists of four rays. If $\lambda_{1}>0>-\lambda_{2}$ are the eigenvalues of $\widehat{M}$ then, with the usual meaning of $R$ 


\begin{tabular}{||c||c|c|c|c|c||}
\hline \hline$n$ & 3 & 4 & 5 & 6 & 7 \\
\hline DQC & 0.659 & 0.348 & 0.167 & 0.075 & 0.035 \\
\hline QC & 0.247 & 0.028 & 0.0013 & 0.00002 & - \\
\hline \hline
\end{tabular}

Table 1: Frequencies of Directionally Quasi-Convex (DQC) and of Quasi-Convex (QC) pairs $(\Omega, M) \in$ $\mathbb{R}^{n} \times \mathrm{LS}_{n}$ for different values of $n$. The result was obtained by applying the DQC algorithm to several hundreds of thousands of pairs, the entries of which are pseudorandom numbers in the interval $(-1,1)$. QC was instead tested over several millions of such pairs; no QC pair was found for $n=7$.

and $Q$, each of the four points

$$
\pm R\left(\begin{array}{c}
Q \bar{y}_{ \pm} \\
0
\end{array}\right), \quad \bar{y}_{ \pm}=\left(\begin{array}{c}
\lambda_{2}^{1 / 2} \\
\pm \lambda_{1}^{1 / 2}
\end{array}\right)
$$

belongs to one of these rays. (These points are constructed in the same way as $\bar{x}$ for $m>3$, but considering all possible choices of signs.) Thus $(\widehat{\Omega}, \widehat{M})$ is DQC if and only none of these points belong to $\mathbb{R}_{+}^{3}$.

B. A concluding comment. We have applied the algorithm to some problems with four and five degrees of freedom, including the Riemann ellipsoids already studied in [7] [8] and the Levitron. In addition, we have also tested some sample problems in higher dimensions. Our impression is that the algorithm performs quite efficiently. For systems with four degrees of freedom it is significantly faster than the one used in [7].

Needless to say, the algorithm becomes less and less efficient for large $n$ : the recursive procedure is called up to $\sum_{k=4}^{n} n ! / k$ ! times, the three-dimensional algorithm is called up to $n ! / 6$ times and, in addition, the invidual operations become more costly. We do not know if this can be improved.

However, the fact that we would like to stress here is that DQC is probably not significant for very large $n$, so that the complexity of the algorithm might not be an issue. One reason is that all estimates provided by the existing theorems on Nekhoroshev-stability depend critically on the number of degrees of freedom and become meaningless when $n \rightarrow \infty$. For instance, typical values of the constants $a$ and $b$ appearing in (1.1) are $a=b=\frac{1}{n}$. Thus, the concept of Nekhoroshev stability itself seems to become less and less significant for large $n$. But in addition, if one thinks of the geometry of DQC, then one realizes that - in purely statistical terms - DQC becomes less and less likely for large $n$.

Any statement on random matrices is delicate, so this matter would require a careful analysis which goes beyond the purposes of the present work. Nevertheless, we have applied the DQC algorithm to several hundreds of thousands of random pairs $(\Omega, M) \in \mathbb{R}^{n} \times \mathrm{LS}_{n}$ for the first few values of $n$. The result is shown in Table 1 and seems to indicate that the "probability of DQC" tends to zero approximately as $2^{-n}$. The Table also shows the numerically computed "probability" that a pair $(\Omega, M)$ be Quasi-Convex. It should be noted that the "probability" of DQC quickly becomes much larger than that of Quasi-Convexity. Thus, it appears that DQC is a valuable generalization of Quasi-Convexity and is probably of practical importance for system with not too many degrees of freedom. 


\section{An example: The Levitron}

The Levitron consists of a symmetric magnetic spinning top levitating in the air over a repelling base magnet. Neglecting friction, it is modelled as a Hamiltonian system with six degrees of freedom. This system was first analysed in an adiabatic approximation in [3]. Conditions for linear stability of the periodic orbit of the pure spinning motion were derived in the exact treatment of [4]. For certain combinations of the structural parameters and of the spin rate this periodic orbit is elliptic, a necessary condition for levitation. Reduction with respect to the body $S^{1}$-symmetry reduces this periodic orbit to an equilibrium of a system with five degrees of freedom [5]. It can be shown that it is not possible to construct a Lyapunov function for this equilibrium with the known integrals of the system. Hence the question arises whether this equilibrium is DQC, and hence Nekhoroshevstable. Here we just give the reduced Hamiltonian and show some results of the DQC analysis for a disk-shaped magnet of assigned structural parameters. Note that this analysis is made here only with the intent of showing an application of the algorithm developed in this article. Its results may or may not be relevant for the behaviour of a real Levitron. This question deserves further investigation.

The reduced Hamiltonian of the Levitron has the form $H=K+U$. We use coordinates $\left(x, y, z, a_{1}, a_{2}\right)$, where $(x, y, z)$ are the coordinates of the center of mass of the top, suitably rescaled, and $\left(a_{1}, a_{2}\right)$ are horizontal coordinates of the unit vector in the direction of the tip of the symmetry axis of the top. The $z$-axis points upward. Denoting by $\left(p_{x}, p_{y}, p_{z}, p_{1}, p_{2}\right)$ the conjugate momenta, the kinetic energy $K$ and the potential energy $U$ are given by

$$
\begin{aligned}
2 K & =p_{x}^{2}+p_{y}^{2}+p_{z}^{2}+p_{1}^{2}+\left(p_{2}-\frac{a_{1} a_{3}}{1-a_{1}^{2}}\right)^{2}-\left(p_{1} a_{1}+p_{2} a_{2}\right)^{2}+\frac{a_{1}^{2} a_{2}^{2}}{\left(1-a_{1}^{2}\right)^{2}} \\
U & =\alpha \beta \gamma z-\frac{\beta}{\gamma} a \cdot B(x, y, z)
\end{aligned}
$$

where $a=\left(a_{1}, a_{2}, a_{3}\right)$ with $a_{3}$ standing for $\sqrt{1-a_{1}^{2}-a_{2}^{2}}$. The parameters $\alpha, \beta$ and $\gamma$ are given by

$$
\alpha=\frac{m g}{\mu}, \quad \beta=\frac{m \mu}{L^{2}}, \quad \gamma=\sqrt{\frac{\Theta}{m}}
$$

where $m, \Theta, \mu$ and $L$ are mass, momentum of inertia, dipole strength and spin rate of the the top, and $g$ is gravity. The magnetic field $B$ is determined by a potential $F$ so that $B=-\nabla F$. In turn $F$ is determined by the field strength on the symmetry axis $\phi(z)$ by

$$
F(x, y, z)=\sum_{j=0}^{\infty} \frac{(-1)^{j}}{\left(2^{j} j !\right)^{2}}\left(x^{2}+y^{2}\right)^{j} \frac{d^{2 j} \phi}{d z^{2 j}}(z) .
$$

The pure spinning motion of the top corresponds to the equilibrium of the reduced system at which all variables except $z$ are zero. The equilibrium value of $z$ is determined by the condition $\partial U / \partial z=0$. As it turns out, the ellipticity is determined by three dimensionless parameteres, which are combinations of $\alpha, \beta, \gamma$ and of the values at the origin of three derivatives of the function $\phi$, see [4] for details.

After a Taylor expansion about the equilibrium, the Hamiltonian is in a form that can be subjected to the normal form computation as described in the appendix. The result of the normal form computation are the vector $\Omega$ and the matrix $M$. As it turns out, these quantities depend 

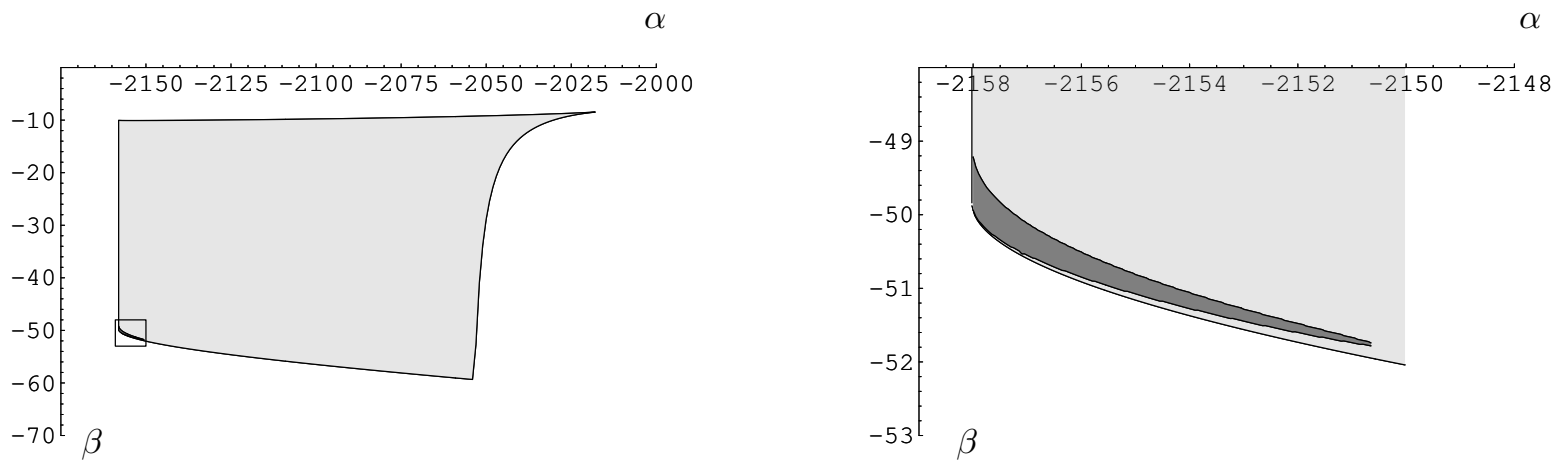

Figure 3: The ellipticity region (light shading) and the DQC region (dark shading) for the Levitron with disk-shaped base magnet described in the text. The figure on the right is an enlargement of the rectangle on the left.

on five parameters, the previous three plus two more determined by two higher order derivatives of $\phi$. A complete investigation of the DQC of the Levitron would therefore require the scanning of a five-dimensional parameter space.

Here, for simplicity, we consider only a specific case. We assume that the base magnet has the shape of a disk of radius $R$, in which case

$$
\phi(z)=2 \pi\left(1-\frac{z}{\sqrt{R^{2}+z^{2}}}\right) .
$$

Moreover, we fix all structural parameters but the mass $m$ and the spin rate $L$, varying correspondingly the momentum of inertia $\Theta$ in such a way that $\gamma=\sqrt{\Theta / m}$ remains constant. Specifically, we consider a situation where $R=0.05$ and $\gamma=0.0075$. The resulting ellipticity region in the plane $(\alpha, \beta)$ is shown in Figure 3. The Birkhoff normal form has been numerically constructed on a grid within such an ellipticity region, and it has then been tested for DQC with the algorithm proposed. The result is that there is only a very small region for which the equilibrium is DQC, see Figure 3. Since this region is extremely small it can probably not be responsible for the observed stability of the Levitron. Further investigation is needed to explore the five-dimensional parameter space of magnets.

\section{A Construction of the Birkhoff normal form}

For the reader's convenience, we describe here the construction of the fourth-order Birkhoff normal form, following closely [7], Section 5. Consider a real analytic Hamiltonian system with $n$ degrees of freedom which has an equilibrium point. In local symplectic coordinates $\xi=\left(q_{1}, \ldots, q_{n}, p_{1}, \ldots, p_{n}\right)$ centered on the equilibrium the Hamiltonian takes the form

$$
h=h_{2}+h_{3}+h_{4}+\ldots
$$

where $h_{k}(\xi)$ is a homogeneous polynomial of degree $k$. Let $X$ be the linearization of the Hamiltonian vector field of $h$ at the equilibrium, that is, the Hamiltonian vector field of $h_{2}$ :

$$
X=\mathbb{J}\left(\begin{array}{l}
\frac{\partial h}{\partial p} \\
\frac{\partial h}{\partial q}
\end{array}\right), \quad \mathbb{J}=\left(\begin{array}{cc}
\mathbf{0}_{n} & \mathbf{1}_{n} \\
-\mathbf{1}_{n} & \mathbf{0}_{n}
\end{array}\right) .
$$


The equilibrium is elliptic if the matrix $X$ has purely imaginary, nonzero eigenvalues $\pm i \omega_{1}, \ldots, \pm i \omega_{n}$; by convention $\omega_{j}>0$.

The first step in the construction of the Birkhoff normal form is the diagonalization of the quadratic term $h_{2}$ by a linear symplectic transformation $T$. If the eigenvalues of $X$ are all distinct, then there is a linear canonical change of coordinates

$$
\xi=\left(\begin{array}{c}
q \\
p
\end{array}\right) \mapsto \Xi=\left(\begin{array}{c}
Q \\
P
\end{array}\right)=T^{-1} \xi
$$

such that $\mathcal{H}_{2}(\Xi):=h_{2}(T \Xi)$ has the form

$$
\mathcal{H}_{2}(Q, P)=\sum_{j=1}^{n} s_{j} \omega_{j} I_{j}\left(Q_{j}, P_{j}\right), \quad I_{j}=\frac{P_{j}^{2}+Q_{j}^{2}}{2},
$$

where each $s_{j}$ is either +1 or -1 . The matrix $T$ and the "Krein signatures" $s_{j}$ are constructed as follows: For $j=1, . ., n$, let $x_{j}^{ \pm}=x_{j}^{\prime} \pm i x_{j}^{\prime \prime}$, with $x_{j}^{\prime}, x_{j}^{\prime \prime} \in \mathbb{R}^{2 n}$, denote an eigenvector of the matrix $X$ associated to the eigenvalue $\pm i \omega_{j}$. It turns out that the numbers

$$
\Gamma_{j}:=x_{j}^{\prime} \cdot \mathbb{J} x_{j}^{\prime \prime}
$$

are nonzero. Define $\left(u_{j}, v_{j}\right)=\left(x_{j}^{\prime}, x_{j}^{\prime \prime}\right)$ if $\Gamma_{j}>0$ and $\left(u_{j}, v_{j}\right)=\left(x_{j}^{\prime \prime}, x_{j}^{\prime}\right)$ if $\Gamma_{j}<0$. Then the matrix $\Gamma$ and the numbers $s_{j}$ are given by

$$
T=\left(\frac{u_{1}}{\gamma_{1}}, \ldots, \frac{u_{n}}{\gamma_{n}}, \frac{v_{1}}{\gamma_{1}}, \ldots, \frac{v_{n}}{\gamma_{n}}\right)^{T} \quad \text { and } \quad s_{j}=\operatorname{Sign}\left(\Gamma_{j}\right),
$$

where $\gamma_{j}=\sqrt{\left|\Gamma^{j}\right|}$; the notation means that the $2 n$ vectors are the rows of the matrix. Note that the condition that all the eigenvalues of $X$ be distinct (and hence nonzero) can be regarded as the condition that the "frequency vector"

$$
\Omega=\left(s_{1} \omega_{1}, \ldots, s_{n} \omega_{n}\right)
$$

has no resonances of order one or two:

$$
\Omega \cdot \nu \neq 0 \quad \text { for all } \nu \in \mathbb{Z}^{n} \quad \text { such that }|\nu|=1,2,
$$

where, for integer vectors, $|\nu|=\left|\nu_{1}\right|+\cdots+\left|\nu_{n}\right|$.

For the construction of the Birkhoff normal form we use complex canonical coordinates $\zeta=$ $\left(w_{1}, \ldots, w_{n}, z_{1}, \ldots, z_{n}\right)$ defined by

$$
w_{j}=-\frac{i Q_{j}+P_{j}}{\sqrt{2}}, \quad z_{j}=\frac{Q_{j}+i P_{j}}{\sqrt{2}}, \quad(j=1, \ldots, n) .
$$

Hence $\Xi=S \zeta$ with

$$
S=\frac{1}{\sqrt{2}}\left(\begin{array}{cc}
i \mathbf{1}_{n} & \mathbf{1}_{n} \\
-\mathbf{1}_{n} & -i \mathbf{1}_{n}
\end{array}\right)
$$

Thus, the Hamiltonian in terms of the complex coordinates $\zeta$ is $H(\zeta)=h(T S \zeta)$ and its quadratic part is $\mathcal{H}(S \zeta)$ which gives

$$
H_{2}=\sum_{j=1}^{n} i \Omega_{j} z_{j} w_{j}
$$


We now describe the construction of the Birkhoff normal form of order four of the Hamiltonian $H$. Given a function $f(w, z)$ denote its Taylor series by

$$
f(w, z)=\sum_{j, k \in \mathbb{N}^{n}} f_{j k} w^{j} z^{k}
$$

where $w^{j}=w_{1}^{j_{1}} \cdots w_{n}^{j_{n}}$ etc. For any integer vector $\nu \in \mathbb{Z}^{n}$ define the $\nu$-th harmonic of $f$ by

$$
\langle f\rangle_{\nu}(w, z)=\sum_{\substack{j, k \in \mathbb{N} n \\ j-k=\nu}} f_{j k} w^{j} z^{k},
$$

so that $f=\sum_{\nu \in \mathbb{Z}^{n}}\langle f\rangle_{\nu}$. The average of $f$ is its harmonic $\langle f\rangle_{0}$. The spectrum of $f$ is $\operatorname{Sp}(f)=\{\nu \in$ $\left.\mathbb{Z}^{n}:\langle f\rangle_{\nu} \neq 0\right\}$.

We construct the Birkhoff normal form by means of the so-called Lie method. Let $\Phi^{\chi}$ denote the time-one-map of the flow of the Hamiltonian vector field of a function $\chi$; the Lie transform generated by $\chi$ of a function $f$ is $f \circ \Phi^{\chi}$. If $f$ and $\chi$ are analytic in an open neighborhood of a point (the equilibrium point, in our case), then $f \circ \Phi^{\chi}$ is also analytic in some sufficiently small, but nonempty, open neighborhood of such a point and there one has $f \circ \Phi^{\chi}=\sum_{j=0}^{\infty} \frac{1}{j !} L_{\chi}^{j} f$. We adopt the sign convention $L_{f} g=\{f, g\}=\frac{\partial f}{\partial z} \frac{\partial g}{\partial w}-\frac{\partial g}{\partial z} \frac{\partial f}{\partial w}$ for the Poisson brackets.

The generating function $\chi$ of the Lie transform is constructed by solving the so-called homological equation, which has the form $\left\{H_{2}, \chi\right\}=f-\langle f\rangle_{0}$ for some function $f$. The solution of this equation is formally given by $\chi=\mathcal{S}_{\Omega}(f)$, where

$$
\mathcal{S}_{\Omega}(f)=\sum_{\nu \in \operatorname{Sp}(f) \backslash\{0\}} \frac{\langle f\rangle_{\nu}}{i \Omega \cdot \nu} .
$$

This is well defined if $\Omega$ does not resonate with any $\nu \in \operatorname{Sp}(f)$ and if the series converges. Here $f$ will always be a polynomial, either $f=H_{3}$ or $f=H_{4}^{\prime}$ (see below), so no convergence problems exist.

The fourth-order Birkhoff normal form of $H=H_{2}+H_{3}+H_{4}+\ldots$, if it exists, is constructed using two Lie transforms, which average the terms of degree three and four of $H$, respectively. The first Lie transform is generated by the solution

$$
\chi_{1}=\mathcal{S}_{\Omega}\left(H_{3}\right)
$$

of the homological equation $\left\{H_{2}, \chi_{1}\right\}=H_{3}-\left\langle H_{3}\right\rangle_{0}$ and is well defined if

$$
\Omega \cdot \nu \neq 0 \quad \forall \nu \in \operatorname{Sp}\left(H_{3}\right) .
$$

Since $\left\langle H_{3}\right\rangle_{0}=0$ the homological equation gives $L_{\chi_{1}}^{2} H_{2}=-L_{\chi_{1}} H_{3}$. This Lie transform conjugates $H=H_{2}+H_{3}+H_{4}+\ldots$ to

$$
H^{\prime}=H_{2}+H_{4}^{\prime}+\ldots, \quad \text { where } \quad H_{4}^{\prime}=\frac{1}{2} L_{\chi_{1}} H_{3}+H_{4} .
$$

The second Lie transform is generated by the solution $\chi_{2}=\mathcal{S}_{\Omega}\left(H_{4}^{\prime}\right)$ of $\left\{H_{2}, \chi_{2}\right\}=H_{4}^{\prime}-\left\langle H_{4}^{\prime}\right\rangle_{0}$. It is well-defined if

$$
\Omega \cdot \nu \neq 0 \quad \forall \nu \in \operatorname{Sp}\left(H_{4}^{\prime}\right) \backslash\{0\}
$$


and leads to the fourth-order Birkhoff normal form

$$
H^{\prime \prime}=H_{2}+H_{4}^{\prime \prime}+\ldots \quad \text { with } \quad H_{4}^{\prime \prime}=\left\langle\frac{1}{2} L_{\chi_{1}} H_{3}+H_{4}\right\rangle_{0} .
$$

For our purposes, the only important points are controlling the existence of the fourth-order Birkhoff normal form and testing that it possesses the convexity properties discussed below. Neither of these tasks require the actual computation of the generator $\chi_{2}$ : one needs only test (A.4) and compute $H_{4}^{\prime \prime}$.

Acknowledgments. This work was done under the auspices of, and was partially funded by, the EU project HPRN-CT-2000-0113 MASIE - Mechanics and Symmetry in Europe. The authors would like to thank Andrea Giacobbe for useful conversations. The second author would also like to thank Ernst Hairer for his hospitality at the Section de Mathématique of the Université de

Genève, where part of this work was done; this visit was made possible by the Swiss National Science Foundation, under grant 20-64954.01.

\section{References}

[1] G. Benettin, F. Fassò, and M. Guzzo, Nekhoroshev-stability of L4 and L5 in the spatial restricted three-body problem. Reg. and Chaotic Dyn. 3, 56-72 (1998)

[2] G. Benettin and G. Gallavotti, Stability of motions near resonances in quasi-integrable Hamiltonian systems. J. Stat. Phys. 44, 293-338 (1986).

[3] M.V. Berry, The Levitron: an adiabatic trap for spins, Proc. Royal Society of London, A 452 1207-1220 (1996)

[4] H.R. Dullin and R.W. Easton, Stability of Levitrons. Physica D 126, 1-17 (1999).

[5] H.R. Dullin and R.W. Easton, Stability of Levitrons. Z. Angew. Math. Mech., 79, 167-170 (1999).

[6] F. Fassò, M. Guzzo and G. Benettin, Nekhoroshev-stability of elliptic equilibria of Hamiltonian systems. Comm. Math. Phys. 197, 347-360 (1998).

[7] F. Fassò and D. Lewis, Stability properties of the Riemann ellipsoids. Arch. Rat. Mech. Anal. 158, 259-292 (2001).

[8] F. Fassò and D. Lewis, Errata: Stability properties of the Riemann ellipsoids. In preparation.

[9] A. Giorgilli, Rigorous results on the power expansions for the integrals of a Hamiltonian system near an elliptic equilibrium point. Ann. Inst. H. Poincaré - Phys. Thèor. 48, 423-439 (1988)

[10] A. Giorgilli, A. Delshams, E. Fontich, L. Galgani and C. Simó, Effective Stability for a Hamiltonian System near an Elliptic Equilibrium Point, with an Application to the Restricted three Body Problem. J. Diff. Eq. 77, 167-198 (1989)

[11] M. Guzzo, F. Fassò and G. Benettin, On the stability of elliptic equilibria. MPEJ - Mathematical Physics Electronic Journal 4, Paper 1 (1998)

[12] P. Lochak, Canonical perturbation theory via simultaneous approximation. Russ. Math. Surv. 47, 57-133 (1992)

[13] K.R. Meyer and G.R. Hall, Introduction to Hamiltonian Dynamical Systems and the N-Body Problem (Springer, New York, 1991). 
[14] N.N. Nekhoroshev, An exponential estimate of the time of stability of nearly integrable hamiltonian systems. Usp. Mat. Nauk 32, 5-66 (1977).

[15] L. Niederman, Nonlinear stability around an elliptic equilibrium point in a Hamiltonian system. Nonlinearity 11, 1465-1479 (1965) 